\title{
THE LEFSCHETZ CONDITION ON PROJECTIVIZATIONS OF COMPLEX VECTOR BUNDLES
}

\author{
Hirokazu Nishinobu and Toshiniro Yamaguchi
}

\begin{abstract}
We consider a condition under which the projectivization $P\left(E^{k}\right)$ of a complex $k$-bundle $E^{k} \rightarrow M$ over an even-dimensional manifold $M$ can have the hard Lefschetz property, affected by [10]. It depends strongly on the rank $k$ of the bundle $E^{k}$. Our approach is purely algebraic by using rational Sullivan minimal models [5]. We will give some examples.
\end{abstract}

\section{Introduction}

A Poincaré duality space $Y$ of the formal dimension

$$
f d(Y)=\max \left\{i ; H^{i}(Y ; \mathbb{Q}) \neq 0\right\}=2 m
$$

is said to be cohomologically symplectic (c-symplectic) if $u^{m} \neq 0$ for some $u \in H^{2}(Y ; \mathbb{Q})$ and, furthermore, is said to have the hard Lefschetz property (or simply the Lefschetz property) with respect to the c-symplectic class $u$, if the maps

$$
\cup u^{j}: H^{m-j}(Y ; \mathbb{Q}) \rightarrow H^{m+j}(Y ; \mathbb{Q}), \quad 0 \leq j \leq m
$$

are monomorphisms (then called the Lefschetz maps) [17]. For example, a compact Kähler manifold has the hard Lefschetz property [17], [6, Theorem 4.35]. Recall the Thurston-Weinstein problem [17, p. 198]: "Describe symplectic compact manifolds with no Kähler structure". Conversely, what conditions on a symplectic manifold imply the existence of a Kähler structure or, more generally, that the manifold satisfies the hard Lefschetz property?

Let $M$ be an even-dimensional manifold and $\xi: E^{k} \rightarrow M$ be a complex $k$-bundle over $M$. The projectivization of the bundle $\xi$

$$
P(\xi): \mathbb{C} P^{k-1} \stackrel{j}{\rightarrow} P\left(E^{k}\right) \rightarrow M
$$

satisfies the rational cohomology algebra condition $(*)$ :

$H^{*}\left(P\left(E^{k}\right) ; \mathbb{Q}\right)=H^{*}(M ; \mathbb{Q})[x] /\left(x^{k}+c_{1} x^{k-1}+\cdots+c_{k-j} x^{j}+\cdots+c_{k-1} x+c_{k}\right)$

Received May 16, 2014; Revised August 28, 2014.

2010 Mathematics Subject Classification. 55P62, 57R17.

Key words and phrases. projectivization, c-symplectic, the Lefschetz property, Sullivan model, formal, projective (n)-Lefschetz, projective non-Lefschetz. 
where $c_{i}$ are the $i$-th Chern classes of $\xi$ and $x$ is a degree 2 class generating the cohomology of the complex projective space fiber (Leray-Hirsch theorem) [3], [10], [17, p. 122]. The manifold $P\left(E^{k}\right)$ appears as the exceptional divisor in blow-up construction for a certain embedding of $M$ [11], [17, Chap. 4]. When $M$ is a non-toral symplectic nilmanifold of dimension $2 n$, there is a bundle $E^{n}$ such that $P\left(E^{n}\right)$ is not Lefschetz [18], [10, Example 4.4]. In general, for a $2 k$-dimensional manifold $M$ and a fibration $\mathbb{C} P^{k-1} \rightarrow E \rightarrow M$, the total space $E$ is Lefschetz if and only if $M$ is Lefschetz [10, Remark 4.2]. We consider the following:

Problem 1.1. Suppose that the projectivization $P\left(E^{k}\right)$ of a $k$-dimensional vector bundle $E^{k} \rightarrow M$ is c-symplectic with respect to $\tilde{x}$ where $j^{*}(\tilde{x})=x$; i.e., $\tilde{x}^{m} \neq 0$ when $\operatorname{dim} P\left(E^{k}\right)=2 m$. What rational homotopical conditions on $M$ are necessary for $P\left(E^{k}\right)$ to have the Lefschetz property with respect to $\tilde{x}$ ?

Proposition 1.2. Let $M$ be an even dimensional manifold.

(1) For a sufficiently large $k$, there is a $k$-dimensional vector bundle $E^{k} \rightarrow M$ such that $P\left(E^{k}\right)$ is c-symplectic with respect to $x$.

(2) If $P\left(E^{k}\right)$ is c-symplectic with respect to $x$, then there is a vector bundle $E^{m} \rightarrow M$ such that $P\left(E^{m}\right)$ is c-symplectic with respect to $x$ for any $m>k$.

Definition 1.3. An even-dimensional manifold (or more general Poincaré duality space) $M$ is said to be projective $(k)$-Lefschetz if there exists a complex $k$-bundle $E^{k}$ such that the projectivization $P\left(E^{k}\right)$ is c-symplectic with respect to $\tilde{x}$ and has the Lefschetz property with respect to $\tilde{x}$. Then we often say simply that $M$ is projective Lefschetz. In particular, we say that $M$ is projective non-Lefschetz if $P\left(E^{k}\right)$ cannot have the Lefschetz property for any $k$ and $E^{k}$.

In this paper, we recall D.Sullivan's rational model in $\S 2$ and we give some examples that indicate how the rational cohomology algebra of $M$ determines the projective $(n)$-Lefschetzness of $M$ when $M$ is the product of at most four spheres in $\S 3$.

Acknowledgement. The authors would like to thank Shoji Yokura for his valuable comments on an earlier version of the paper and the referee for many helpful suggestions.

\section{Sullivan model}

Let $\mathcal{M}(Y)=(\Lambda V, d)$ be the Sullivan minimal model of a nilpotent space $Y$. It is a freely generated $\mathbb{Q}$-commutative differential graded algebra (abbr. DGA) with a $\mathbb{Q}$-graded vector space $V=\bigoplus_{i>1} V^{i}$ where $\operatorname{dim} V^{i}<\infty, V$ admits a basis $\left\{v_{\alpha}\right\}$ indexed by a well-ordered set $\{\alpha\} \operatorname{such}$ that $\operatorname{deg}\left(v_{\alpha}\right) \leq \operatorname{deg}\left(v_{\beta}\right)$ if $\alpha<\beta$ and $d\left(v_{\alpha}\right) \in \Lambda\left(v_{\beta}\right)_{\beta<\alpha}$. The differential $d$ is a decomposable; i.e., $d\left(V^{i}\right) \subset\left(\Lambda^{+} V \cdot \Lambda^{+} V\right)^{i+1}$. Here $\Lambda^{+} V$ is the ideal of $\Lambda V$ generated by elements of positive degree. Denote the degree of a homogeneous element $f$ of a graded algebra as $|f|$. Then $x y=(-1)^{|x||y|} y x$ and $d(x y)=d(x) y+(-1)^{|x|} x d(y)$. Note 
that $\mathcal{M}(Y)$ determines the rational homotopy type of $Y$. In particular, it is known that

$$
H^{*}(\Lambda V, d) \cong H^{*}(Y ; \mathbb{Q}) \text { and } V^{i} \cong \operatorname{Hom}\left(\pi_{i}(Y), \mathbb{Q}\right) .
$$

See $[5, \S 12 \sim \S 15]$ for details. When $\pi_{*}(Y) \otimes \mathbb{Q}<\infty$ and $\operatorname{dim} H^{*}(Y ; \mathbb{Q})<\infty, Y$ is said to be elliptic. It is known that

$$
f d(Y)=f d(\Lambda V, d)=\sum_{i}\left|y_{i}\right|-\sum_{i}\left(\left|x_{i}\right|-1\right)
$$

for $V^{\text {odd }}=\mathbb{Q}\left(y_{i}\right)_{i}$ and $V^{\text {even }}=\mathbb{Q}\left(x_{i}\right)_{i}$ when $Y$ is elliptic [5, $\left.\S 32\right]$.

Proposition 2.1. Let $M$ be an even dimensional manifold. Then there is a graded algebra $A=H^{*}(M ; \mathbb{Q})[x] /\left(x^{k}+c_{1} x^{k-1}+\cdots+c_{k-j} x^{j}+\cdots+c_{k-1} x+c_{k}\right)$ with $|x|=2$ and $c_{i} \in H^{2 i}(M ; \mathbb{Q})$ if and only if there is a complex $k$-bundle $\xi: E^{k} \rightarrow M$ such that $c_{i}$ are the Chern classes of $\xi$ by suitable scalar multiplying and $A$ is the rational cohomology of $P\left(E^{k}\right)$.

Proof. The set of equivalence classes of complex $k$-vector bundles over $M$ is identified as the homotopy set from $M$ to the complex Grassmanian $G(k, N)$ of $k$-planes in $\mathbb{C}^{N}$ for a sufficiently large $N[2, \mathrm{IV}]$. Then the Chern classes of a $k$-bundle are given as $f^{*}\left(c_{1}(\gamma)\right), \ldots, f^{*}\left(c_{k}(\gamma)\right)$ for the classifying map $f$ and the universal bundle $\gamma$ over $G(k, N)$. Conversely, for given elements $c_{1}, \ldots, c_{k}$, a rational map $M \rightarrow M_{(0)} \rightarrow G(k, N)_{(0)}$ induced by $\Pi_{i} c_{i}: M \rightarrow \Pi_{i} K(\mathbb{Q}, 2 i) \simeq$ $B U(k)_{(0)}$ is factored through a map $f: M \rightarrow G(k, N)$ [12, Theorem 5.3] because $G(k, N)=U(N) / U(k) \times U(N-k)$ is 0-universal [1, Proposition 3.7]. Here $B U(k)$ is the classifying space of the unitary group $U(k)$ and $Y_{(0)}$ is the rationalization of a space $Y$ [8]. Thus we obtain the appropriate $k$-bundle as the pullback of $\gamma$ by $f$.

Corollary 2.2. The projective Lefschetzness of an even-dimensional manifold $M$ depends only on the graded algebra $H^{*}(M ; \mathbb{Q})$.

Let $\mathcal{M}\left(\mathbb{C} P^{k-1}\right)=(\mathbb{Q}[x] \otimes \Lambda(y), d)$ with $d(y)=x^{k}$ and $d(x)=0$. From Corollary 2.2, the information of $P\left(E^{k}\right)$ that we need in this note is given as the relative Sullivan model $[5, \S 14]$ :

$$
\left(H^{*}(M ; \mathbb{Q}), 0\right) \rightarrow\left(H^{*}(M ; \mathbb{Q}) \otimes \mathbb{Q}[x] \otimes \Lambda(y), D\right) \rightarrow(\mathbb{Q}[x] \otimes \Lambda(y), d)
$$

with $D(f)=0$ for $f \in H^{*}(M ; \mathbb{Q}), D(x)=0$ and

$$
D(y)=x^{k}+c_{1} x^{k-1}+\cdots+c_{k-j} x^{j}+\cdots+c_{k-1} x+c_{k},
$$

where $c_{i} \in H^{2 i}(M ; \mathbb{Q})$ are the Chern classes of $\xi$. Especially, we don't need the assumption that $M$ is nilpotent. Remark that $H^{*}\left(P\left(E^{k}\right) ; \mathbb{Q}\right) \cong H^{*}\left(H^{*}(M ; \mathbb{Q})\right.$ $\otimes \mathbb{Q}[x] \otimes \Lambda(y), D)$ as a $\mathbb{Q}$-graded algebra and then

$$
H^{j}\left(P\left(E^{k}\right) ; \mathbb{Q}\right)=H^{j}(M ; \mathbb{Q}) \oplus H^{j-2}(M ; \mathbb{Q}) x \oplus \cdots \oplus H^{j-2 k+2}(M ; \mathbb{Q}) x^{k-1} .
$$

Notice that $(* *)$ is equivalent to $(*)$ of $\S 1$ and also equivalent to

$$
\left[x^{k}\right]=-\left[c_{1} x^{k-1}+\cdots+c_{k-j} x^{j}+\cdots+c_{k-1} x+c_{k}\right]
$$


in $H^{*}\left(P\left(E^{k}\right) ; \mathbb{Q}\right)$, which is the only relation between the elements of $H^{*}(M ; \mathbb{Q})$ and $x$. Then, for example, $\left[x^{k+1}\right]=-\left[c_{1} x^{k}+\cdots+c_{k-j} x^{j+1}+\cdots+c_{k-1} x^{2}+\right.$ $\left.c_{k} x\right]=\left[c_{1}^{2} x^{k-1}+\cdots+\left(c_{1} c_{k-j}-c_{k-j+1}\right) x^{j}+\cdots+\left(c_{1} c_{k-1}-c_{k}\right) x+c_{1} c_{k}\right]$. In particular,

$(* * *) \quad[a] \neq 0$ in $H^{*}(M ; \mathbb{Q})$ if and only if $\left[a x^{j}\right] \neq 0$ in $H^{*}\left(P\left(E^{k}\right) ; \mathbb{Q}\right)$

for any $0 \leq j<k$.

Lemma 2.3. Let $A=\left(H^{*}(M ; \mathbb{Q}) \otimes \mathbb{Q}[x] \otimes \Lambda(y), D\right)$ with $D(y)=x^{k}+c_{1} x^{k-1}+$ $\cdots+c_{k-1} x+c_{k}$ and let $B=\left(H^{*}(M ; \mathbb{Q}) \otimes \mathbb{Q}[x] \otimes \Lambda\left(y^{\prime}\right), D^{\prime}\right)$ with $D^{\prime}\left(y^{\prime}\right)=$ $x^{m-k} D(y)=x^{m}+c_{1} x^{m-1}+\cdots+c_{k-1} x^{m-k+1}+c_{k} x^{m-k}$ for $k<m$. If $[f] \neq 0$ in $H^{*}(A)$, then $\left[f x^{m-k}\right] \neq 0$ in $H^{*}(B)$.

Proof. Notice that an element of $H^{*}(A)$ is identified as one of $H^{*}(B)$ since $H^{*}(A)$ is a submodule of $H^{*}(B)$ over $H^{*}(M ; \mathbb{Q})$. Suppose that $[f]=\left[a_{1} x^{k-1}+\right.$ $\left.\cdots+a_{k-1} x+a_{k}\right] \neq 0$ in $H^{*}(A)$ for $\left[a_{*}\right] \in H^{*}(M ; \mathbb{Q})$. Then there is an index $i$ with $\left[a_{i}\right] \neq 0$ in $H^{*}(M ; \mathbb{Q})$. Thus, in $H^{*}(B),\left[f x^{m-k}\right]=\left[a_{1} x^{m-1}+\cdots+\right.$ $\left.a_{k-1} x^{m-k+1}+a_{k} x^{m-k}\right]=\left[a_{1}\right] x^{m-1}+\cdots+\left[a_{k-1}\right] x^{m-k+1}+\left[a_{k}\right] x^{m-k} \neq 0$ from $(* * *)$.

Proof of Proposition 1.2. From Proposition 2.1, it is sufficient to construct a certain DGA $\left(H^{*}(M ; \mathbb{Q}) \otimes \mathbb{Q}[x] \otimes \Lambda(y), D\right)$. Let $\operatorname{dim} M=2 n$.

(1) Let $\Omega$ be the fundamental class of $M$. Then we can define $\left(H^{*}(M ; \mathbb{Q}) \otimes\right.$ $\mathbb{Q}[x] \otimes \Lambda(y), D)$ by $D(y)=\Omega x^{k-n}+x^{k}$ for $k \geq n$. Notice $\operatorname{dim} P\left(E^{k}\right)=$ $\operatorname{dim} M+\operatorname{dim} \mathbb{C} P^{k-1}=2 n+2 k-2$. Then we have $\left[x^{n+k-1}\right]=-\left[\left(\Omega x^{k-n}\right) x^{n-1}\right]=$ $-\left[\Omega x^{k-1}\right] \neq 0$ from $(* * *)$.

(2) Suppose that the DGA $\left(H^{*}(M ; \mathbb{Q}) \otimes \mathbb{Q}[x] \otimes \Lambda(y), D\right)$ makes $P\left(E^{k}\right)$ csymplectic; i.e., $\left[x^{n+k-1}\right] \neq 0$. Then, for $m>k$, the DGA $\left(H^{*}(M ; \mathbb{Q}) \otimes \mathbb{Q}[x] \otimes\right.$ $\left.\Lambda\left(y^{\prime}\right), D^{\prime}\right)$ with $\left|y^{\prime}\right|=2 m-1$ and $D^{\prime}\left(y^{\prime}\right):=x^{m-k} D(y)$ makes a $2 n+2 m-$ 2-dimensional manifold $P\left(E^{m}\right)$ c-symplectic. Indeed, $\left[x^{n+m-1}\right]=\left[x^{n+k-1}\right.$. $\left.x^{m-k}\right] \neq 0$ in cohomology from Lemma 2.3.

In (2) in Proposition 1.2 , the bundle $E^{m}$ is geometrically realized as the Whitney sum $E^{k} \oplus \theta^{m-k}$ where $\theta^{m-k}$ is the trivial $m-k$-bundle over $M$, in the manner of Proposition 2.1. Thus, if $P\left(E^{k}\right)$ is c-symplectic with respect to $x$, then $P\left(E^{k} \oplus \theta^{m}\right)$ is c-symplectic with respect to $x$ for any $m>0$.

\section{Examples}

In this section, let $M$ be a 2-connected even-dimensional manifold and $\operatorname{dim} P\left(E^{k}\right)=2 m$.

Theorem 3.1. The $2 n$-dimensional sphere $S^{2 n}$ is projective $(k)$-Lefschetz for any $k \geq n$.

Proof. Let $H^{*}\left(S^{2 n} ; \mathbb{Q}\right)=\mathbb{Q}[v] /\left(v^{2}\right)$ with $|v|=2 n$. Consider $P\left(E^{k}\right)$ such that $\operatorname{dim} P\left(E^{k}\right)=2 m$ and $D(y)=v x^{k-n}+x^{k}$ for $k \geq n$. Then $m=n+k-1$ from $2 m=\operatorname{dim} \mathbb{C} P^{k-1}+\operatorname{dim} S^{2 n}=2 n+2 k-2$. Since $\left[x^{m}\right]=-\left[v x^{k-n}\right.$. 
$\left.x^{m-k}\right]=-\left[v x^{k-1}\right] \neq 0$ from $(* * *), P\left(E^{k}\right)$ is c-symplectic with respect to $x$. Furthermore, $\cup x^{k-n-1-2 i}\left(v x^{i}\right)=v x^{k-n-1-i} \neq 0$ in

$$
\cup x^{k-n-1-2 i}: H^{m-(k-n-1-2 i)}\left(P\left(E^{k}\right) ; \mathbb{Q}\right) \rightarrow H^{m+(k-n-1-2 i)}\left(P\left(E^{k}\right) ; \mathbb{Q}\right)
$$

for $i \geq 0$ Thus $S^{2 n}$ is projective $(k)$-Lefschetz.

Proposition 3.2. When $M$ has the rational homotopy type of the product of odd spheres such that $H^{*}(M ; \mathbb{Q}) \cong \Lambda\left(v_{1}, v_{2}, \ldots, v_{n}\right)$ with all $\left|v_{i}\right|$ odd and $1<\left|v_{1}\right| \leq\left|v_{2}\right| \leq \cdots \leq\left|v_{n}\right|$ ( $n$ even), then there exists a bundle $E^{k}$ such that $P\left(E^{k}\right)$ is c-symplectic if and only if $\left|v_{1}\right|+\left|v_{n}\right| \leq 2 k,\left|v_{2}\right|+\left|v_{n-1}\right| \leq$ $2 k, \ldots,\left|v_{n / 2}\right|+\left|v_{n / 2+1}\right| \leq 2 k$.

Proof. (sketch) The minimal DGA $\left(\mathbb{Q}[x] \otimes \Lambda\left(v_{1}, v_{2}, \ldots, v_{n}, y\right), D\right)$ with $|y|=$ $2 k-1$ is c-symplectic if $D\left(v_{1}\right)=\cdots=D\left(v_{n}\right)=0$ and

$$
D(y)=v_{1} v_{n} x^{a_{1}}+v_{2} v_{n-1} x^{a_{2}}+\cdots+v_{n / 2} v_{n / 2+1} x^{a_{n / 2}}+x^{k}
$$

for $a_{i}=\left(2 k-\left|v_{i}\right|-\left|v_{n-i+1}\right|\right) / 2 \geq 0$. Then we have the "if" part from Proposition 2.1 and [14, Theorem 1.2]. The "only if" part is obvious from [14, Theorem $1.2]$.

Theorem 3.3. Let $M=S^{a} \times S^{b}$ with $a \leq b$.

(i) When $a=b$, it is projective $(k)$-Lefschetz for $k \geq b$.

(ii) When $a$ and $b$ are even, it is projective $\left(\frac{b}{2}\right)$-Lefschetz.

(iii) When $a$ and $b$ are odd with $a<b$, it is projective non-Lefschetz.

Proof. Note that $H^{*}(M ; \mathbb{Q})=\mathbb{Q}\left[v_{1}, v_{2}\right] /\left(v_{1}^{2}, v_{2}^{2}\right)=\mathbb{Q}\left(1, v_{1}, v_{2}, v_{1} v_{2}\right)$ as a $\mathbb{Q}$ graded vector space with $|1|=0,\left|v_{1}\right|=a,\left|v_{2}\right|=b$ and $\left|v_{1} v_{2}\right|=a+b$. Consider $P\left(E^{k}\right)$ such that $\operatorname{dim} P\left(E^{k}\right)=2 m$ and

$$
D(y)=v_{1} v_{2} x^{k-\frac{a+b}{2}}+x^{k}
$$

for $k \geq(a+b) / 2$. Then $m=\frac{a+b}{2}+k-1$ from $2 m=a+b+2 k-2$ and $\cup x^{m-a}\left(v_{1}\right)=v_{1} x^{m-a}=v_{1} x^{\frac{a+b}{2}+k-1-a}$ in

$$
\cup x^{m-a}: H^{a}\left(P\left(E^{k}\right) ; \mathbb{Q}\right) \rightarrow H^{2 m-a}\left(P\left(E^{k}\right) ; \mathbb{Q}\right)
$$

for $0 \leq a \leq m$. In cohomology, this element has the form $v_{1} x^{\geq k}=0$ if and only if $a<b$. Thus, when $a<b, \cup x^{m-a}\left(v_{1}\right)=0$; i.e., $\cup x^{m-a}$ is not the Lefschetz map. On the other hand, when $a=b$, we have from $(* * *)$

$$
\begin{aligned}
& \cup x^{m-2 i}\left(x^{i}\right)=x^{m-i}, \\
& \cup x^{m-a-2 i}\left(v_{1} x^{i}\right)=v_{1} x^{m-a-i}, \\
& \cup x^{m-b-2 i}\left(v_{2} x^{i}\right)=v_{2} x^{m-b-i}, \\
& \cup x^{m-a-b-2 i}\left(v_{1} v_{2} x^{i}\right)=v_{1} v_{2} x^{m-a-b-i},
\end{aligned}
$$

whose linear combination can not be zero in cohomology. Thus $M$ is projective ( $k$ )-Lefschetz for $k \geq b$ when $a=b$. 
Let $a \leq b$ be even. Consider $P\left(E^{k}\right)$ such that $\operatorname{dim} P\left(E^{k}\right)=2 m$ and

$$
D(y)=v_{1} x^{\frac{b-a}{2}}+v_{2}+x^{\frac{b}{2}} . \quad\left(k=\frac{b}{2}\right)
$$

Then $m=\frac{a}{2}+b-1$ and we have from $(* * *)$

$$
\begin{aligned}
& \cup x^{m-2 i}\left(x^{i}\right)=x^{m-i}, \\
& \cup x^{m-a-2 i}\left(v_{1} x^{i}\right)=v_{1} x^{m-a-i}= \begin{cases}v_{1} v_{2} x^{m-a-\frac{b}{2}-i} & \left(i<\frac{b-a}{2}\right) \\
v_{1} x^{m-a-i} & \left(\frac{b-a}{2} \leq i<\frac{-a+2 b}{4}\right),\end{cases} \\
& \cup x^{m-b-2 i}\left(v_{2} x^{i}\right)=v_{2} x^{m-b-i}, \\
& \cup x^{m-a-b-2 i}\left(v_{1} v_{2} x^{i}\right)=v_{1} v_{2} x^{m-a-b-i},
\end{aligned}
$$

whose linear combination can not be zero in cohomology; i.e., $\cup x^{j}$ are the Lefschetz maps. Thus $M$ is projective $\left(\frac{b}{2}\right)$-Lefschetz.

Remark 3.4. Even if $M$ is projective $(k)$-Lefschetz, it is not projective $(m)$ Lefschetz for $m>k$, in general. For example, when $M=S^{4} \times S^{6}, M$ is projective (3)-Lefschetz from Theorem 3.3 but not projective (4)-Lefschetz. Indeed, in the proof of Theorem 3.3, $\cup x^{2}: H^{m-2}\left(P\left(E^{4}\right)\right) \rightarrow H^{m+2}\left(P\left(E^{4}\right)\right)$ is not a monomorphism since $\cup x^{2}\left(\left[v_{1} x+v_{2}+x^{3}\right]\right)=\left[v_{1} x^{3}+v_{2} x^{2}+x^{5}\right]=0$, when $D y=v_{1} x^{2}+v_{2} x+x^{4}(m=8)$.

Theorem 3.5. Let $M=S^{a} \times S^{b} \times S^{c}$ with $a \leq b \leq c$. We have the following:

(i) When $a, b$ and $c$ are even, $M$ is projective $\left(\frac{c}{2}\right)$-Lefschetz.

(ii) When $a$ and $c$ are odd, $b$ is even, $M$ is projective non-Lefschetz.

(iii) When $a$ is even, $b$ and $c$ are odd, $M$ is projective Lefschetz if and only if $b=c$. Then $M$ is projective (b)-Lefschetz.

(iv) When $a$ and $b$ are odd, $c$ is even, $M$ is projective Lefschetz if and only if $a=b$. Then $M$ is projective $\left(\max \left\{a, \frac{c}{2}\right\}\right)$-Lefschetz.

Proof. Then $\operatorname{dim} M=a+b+c$ and $H^{*}(M ; \mathbb{Q})=\Lambda\left(v_{1}, v_{2}, v_{3}\right) /\left(v_{1}^{2}, v_{2}^{2}, v_{3}^{2}\right)$ with $\left|v_{1}\right|=a,\left|v_{2}\right|=b,\left|v_{3}\right|=c$.

(i) When $k=\frac{c}{2}, \operatorname{dim} P\left(E^{k}\right)=a+b+2 c-2$ and $m=\frac{a+b+2 c-2}{2}$. Then $|y|=c-1$ and $d(y)=x^{\frac{c}{2}}$. Let $D(y)=v_{1} x^{\frac{c-a}{2}}+v_{2} x^{\frac{c-b}{2}}+v_{3}+x^{\frac{c}{2}}$. Then $P\left(E^{k}\right)$ is $c$-symplectic by $x$ since $\left[x^{m}\right]=-\left[6 v_{1} v_{2} v_{3} x^{\frac{c-2}{2}}\right] \neq 0$. Moreover, we have from $(* * *)$

$$
\begin{aligned}
& \cup x^{m-2 i}\left(x^{i}\right)=x^{m-i} \text {, } \\
& \cup x^{m-a-2 i}\left(v_{1} x^{i}\right)=\left\{\begin{array}{l}
2 v_{1} v_{2} v_{3} x^{\frac{-a+c-2}{2}-i}\left(0 \leq i<\frac{-a+b}{2}\right) \\
v_{1} v_{2} v_{3} x^{\frac{-a+c-2}{2}-i}-v_{1} v_{3} x^{\frac{-a+b+c-2}{2}-i}\left(\frac{-a+b}{2} \leq i<\frac{-a+c}{2}\right) \\
-v_{1} v_{2} x^{\frac{-a+2 c-2}{2}-i}-v_{1} v_{3} x^{\frac{-a+b+c-2}{2}-i}\left(\frac{-a+c}{2} \leq i<\frac{-a+b+2 c}{4}\right),
\end{array}\right. \\
& \cup x^{m-b-2 i}\left(v_{2} x^{i}\right)=\left\{\begin{array}{l}
v_{1} v_{2} v_{3} x^{\frac{-b+c-2}{2}-i}-v_{2} v_{3} x^{\frac{a-b+c-2}{2}-i}\left(0 \leq i<\frac{-b+c}{2}\right) \\
-v_{1} v_{2} x^{\frac{-b+2 c-2}{2}-i}-v_{2} v_{3} x^{\frac{a-b+c-2}{2}-i}\left(\frac{-b+c}{2} \leq i<\frac{a-b+c}{2}\right) \\
v_{2} x^{\frac{a-b+2 c-2}{2}-i}\left(\frac{a-b+c}{2} \leq i<\frac{a-b+2 c}{4}\right),
\end{array}\right.
\end{aligned}
$$




$$
\begin{aligned}
& \cup x^{m-c-2 i}\left(v_{3} x^{i}\right)=\left\{\begin{array}{l}
-v_{1} v_{3} x^{\frac{b-2}{2}-i}-v_{2} v_{3} x^{\frac{a-2}{2}-i}\left(0 \leq i<\frac{a+b-2}{2}\right) \\
v_{3} x^{\frac{a+b-2}{2}}\left(\frac{a+b-c}{2} \leq i<\frac{a+b}{4}\right),
\end{array}\right. \\
& \cup x^{m-(a+b)-2 i}\left(v_{1} v_{2} x^{i}\right)=\left\{\begin{array}{l}
-v_{1} v_{2} v_{3} x^{\frac{-a-b+c-2}{2}-i}\left(0 \leq i<\frac{-a-b+c}{2}\right) \\
v_{1} v_{2} x^{\frac{-a-b+2 c-2}{2}-i}\left(\frac{-a-b+c}{2} \leq i<\frac{-a-b+2 c}{4}\right),
\end{array}\right. \\
& \cup x^{m-(a+c)-2 i}\left(v_{1} v_{3} x^{i}\right)=v_{1} v_{3} x^{\frac{-a+b-2}{2}-i}(a<b),
\end{aligned}
$$

whose linear combination can not be zero in cohomology. Thus $M$ is projective $\left(\frac{c}{2}\right)$-Lefschetz.

(ii) For $|y|=2 k-1$ and $m=\frac{a+b+c+2 k-2}{2}$, there are two types of $c$-symplectic models as follows:

$$
D(y)=v_{1} v_{3} x^{k-\frac{a+c}{2}}+v_{2} x^{k-\frac{b}{2}}+x^{k} .
$$

Then $\cup x^{m-a}\left(v_{1}\right)=-v_{1} v_{2} x^{\frac{-a+c+2 k-2}{2}}=-v_{1} v_{2} x^{\geq k}=0$ from $a<c$.

$$
D(y)=v_{1} v_{2} v_{3} x^{k-\frac{a+b+c}{2}}+x^{k} .
$$

Then $\cup x^{m-a}\left(v_{1}\right)=v_{1} x^{\frac{-a+b+c+2 k-2}{2}}=v_{1} x^{\geq k}=0$. Thus the Lefschetz maps do not exist in both cases (1) and (2).

(iii) Let $b<c$. For $|y|=2 k-1$ and $m=\frac{a+b+c+2 k-2}{2}$, there are two types of $c$-symplectic models as follows:

$$
D(y)=v_{1} x^{k-\frac{a}{2}}+v_{2} v_{3} x^{k-\frac{b+c}{2}}+x^{k} .
$$

Then $\cup x^{m-b}\left(v_{2}\right)=-v_{1} v_{2} x^{\frac{-b+c+2 k-2}{2}}=-v_{1} v_{2} x^{\geq k}=0$ from $b<c$.

$$
D(y)=v_{1} v_{2} v_{3} x^{k-\frac{a+b+c}{2}}+x^{k} .
$$

Then $\cup x^{m-a}\left(v_{1}\right)=v_{1} x^{\frac{-a+b+c+2 k-2}{2}}=v_{1} x^{\geq k}=0$. Thus the Lefschetz maps do not exist in both cases (1) and (2).

Let $b=c$. Then $M=S^{a} \times S^{b} \times S^{b}$, $\operatorname{dim} M=a+2 b$ and $H^{*}(M ; \mathbb{Q})=$ $\mathbb{Q}\left[v_{1}\right] /\left(v_{1}^{2}\right) \otimes \Lambda\left(v_{2}, v_{3}\right)$ with $\left|v_{1}\right|=a,\left|v_{2}\right|=\left|v_{3}\right|=b$. When $k=b, \operatorname{dim} P\left(E^{k}\right)=$ $a+4 b-2$ and $m=\frac{a+4 b-2}{2}$. Then $|y|=2 b-1$ and $d(y)=x^{b}$. Let $D(y)=$ $v_{1} x^{b-\frac{a}{2}}+v_{2} v_{3}+x^{b}$. Then $P\left(E^{k}\right)$ is $c$-symplectic with respect to $x$. Moreover, we have from $(* * *)$

$$
\begin{aligned}
& \cup x^{m-2 i}\left(x^{i}\right)=x^{m-i}, \\
& \cup x^{m-a-2 i}\left(v_{1} x^{i}\right)=\left\{\begin{array}{l}
-v_{1} v_{2} v_{3} x^{\frac{-a+2 b-2}{2}}-i \\
v_{1} x^{\frac{-a+4 b-2}{2}-i}\left(\frac{-a+2 b}{2} \leq i<\frac{-a+4 b}{4}\right),
\end{array}\right. \\
& \cup x^{m-b-2 i}\left(v_{2} x^{i}\right)=\left\{\begin{array}{l}
-v_{1} v_{2} x^{b-1-i}\left(0 \leq i<\frac{a}{2}\right) \\
v_{2} x^{\frac{a+2 b-2}{2}-i}\left(\frac{a}{2} \leq i<\frac{a+2 b}{4}\right),
\end{array}\right. \\
& \cup x^{m-b-2 i}\left(v_{3} x^{i}\right)=\left\{\begin{array}{l}
-v_{1} v_{3} x^{b-1-i}\left(0 \leq i<\frac{a}{2}\right) \\
v_{3} x^{\frac{a+2 b-2}{2}-i}\left(\frac{a}{2} \leq i<\frac{a+2 b}{4}\right),
\end{array}\right. \\
& \cup x^{m-(a+b)-2 i}\left(v_{1} v_{2} x^{i}\right)=v_{1} v_{2} x^{\frac{-a+2 b-2}{2}-i},
\end{aligned}
$$




$$
\begin{aligned}
& \cup x^{m-(a+b)-2 i}\left(v_{1} v_{3} x^{i}\right)=v_{1} v_{3} x^{\frac{-a+2 b-2}{2}-i}, \\
& \cup x^{m-2 b-2 i}\left(v_{2} v_{3} x^{i}\right)=v_{2} v_{3} x^{\frac{a-2}{2}-i},
\end{aligned}
$$

whose linear combination can not be zero in cohomology. Thus $M$ is projective (b)-Lefschetz. The proof of (iv) is similar to that of (iii).

Theorem 3.6. Let $M=S^{a} \times S^{b} \times S^{c} \times S^{d}$ with $a \leq b \leq c \leq d$. When $a, b, c$ and $d$ are odd, $M$ is projective Lefschetz if and only if $a=b$ and $c=d$. Then $M$ is projective (c)-Lefschetz.

Proof. Let $a<b$. For $|y|=2 k-1$ and $m=\frac{a+b+c+d+2 k-2}{2}$, there are four types of $c$-symplectic models as follows:

$$
D(y)=v_{1} v_{2} x^{k-\frac{a+b}{2}}+v_{3} v_{4} x^{k-\frac{c+d}{2}}+x^{k} .
$$

Then $\cup x^{m-a}\left(v_{1}\right)=-v_{1} v_{3} v_{4} x^{\frac{-a+b+2 k-2}{2}}=-v_{1} v_{3} v_{4} x^{\geq k}=0$.

$$
D(y)=v_{1} v_{3} x^{k-\frac{a+c}{2}}+v_{2} v_{4} x^{k-\frac{b+d}{2}}+x^{k} .
$$

Then $\cup x^{m-a}\left(v_{1}\right)=-v_{1} v_{2} v_{4} x^{\frac{-a+c+2 k-2}{2}}=-v_{1} v_{2} v_{4} x^{\geq k}=0$.

$$
D(y)=v_{1} v_{4} x^{k-\frac{a+d}{2}}+v_{2} v_{3} x^{k-\frac{b+c}{2}}+x^{k} .
$$

Then $\cup x^{m-a}\left(v_{1}\right)=-v_{1} v_{2} v_{3} x^{\frac{-a+d+2 k-2}{2}}=-v_{1} v_{2} v_{3} x^{\geq k}=0$.

$$
D(y)=v_{1} v_{2} v_{3} v_{4} x^{k-\frac{a+b+c+d}{2}}+x^{k} .
$$

Then $\cup x^{m-a}\left(v_{1}\right)=v_{1} x^{\geq k}=0$. Thus, when $a<b, M$ is projective nonLefschetz.

Let $c<d$. For $|y|=2 k-1$ and $m=\frac{a+b+c+d+2 k-2}{2}$, there are four types of $c$-symplectic models as follows:

$$
D(y)=v_{1} v_{2} x^{k-\frac{a+b}{2}}+v_{3} v_{4} x^{k-\frac{c+d}{2}}+x^{k} .
$$

Then $\cup x^{m-c}\left(v_{3}\right)=-v_{1} v_{2} v_{3} x^{\frac{-c+d+2 k-2}{2}}=-v_{1} v_{2} v_{3} x^{\geq k}=0$.

$$
D(y)=v_{1} v_{3} x^{k-\frac{a+c}{2}}+v_{2} v_{4} x^{k-\frac{b+d}{2}}+x^{k} .
$$

Then $\cup x^{m-b}\left(v_{2}\right)=v_{1} v_{2} v_{3} x^{\frac{-b+d+2 k-2}{2}}=v_{1} v_{2} v_{3} x^{\geq k}=0$.

$$
D(y)=v_{1} v_{4} x^{k-\frac{a+d}{2}}+v_{2} v_{3} x^{k-\frac{b+c}{2}}+x^{k} .
$$

Then $\cup x^{m-a}\left(v_{1}\right)=-v_{1} v_{2} v_{3} x^{\frac{-a+d+2 k-2}{2}}=-v_{1} v_{2} v_{3} x^{\geq k}=0$.

$$
D(y)=v_{1} v_{2} v_{3} v_{4} x^{k-\frac{a+b+c+d}{2}}+x^{k} .
$$

Then $\cup x^{m-a}\left(v_{1}\right)=v_{1} x^{\geq k}=0$. Thus, when $c<d, M$ is projective nonLefschetz.

Let $a=b$ and $c=d$. Then $M=S^{a} \times S^{a} \times S^{c} \times S^{c}, \operatorname{dim} M=2 a+2 c$ and $H^{*}(M ; \mathbb{Q})=\Lambda\left(v_{1}, v_{2}, v_{3}, v_{4}\right)$ with $\left|v_{1}\right|=\left|v_{2}\right|=a,\left|v_{3}\right|=\left|v_{4}\right|=c$. When $k=c$, $\operatorname{dim} P\left(E^{k}\right)=2 a+4 c-2$ and $m=a+2 c-1$. Then $|y|=2 k-1$ and $d(y)=x^{c}$. 
Let $D(y)=v_{1} v_{2} x^{c-a}+v_{3} v_{4}+x^{c}$. Then $P\left(E^{k}\right)$ is $c$-symplectic with respect to $x$. Moreover, we have from $(* * *)$

$$
\begin{aligned}
& \cup x^{m-2 i}\left(x^{i}\right)=x^{m-i}, \\
& \cup x^{m-a-2 i}\left(v_{1} x^{i}\right)=-v_{1} v_{3} v_{4} x^{c-1-i}, \\
& \cup x^{m-a-2 i}\left(v_{2} x^{i}\right)=-v_{2} v_{3} v_{4} x^{c-1-i}, \\
& \cup x^{m-c-2 i}\left(v_{3} x^{i}\right)=\left\{\begin{array}{l}
-v_{1} v_{2} v_{3} x^{c-1-i}(0 \leq i<a) \\
v_{3} x^{a+c-1-i}\left(a \leq i<\frac{a+c}{2}\right),
\end{array}\right. \\
& \cup x^{m-c-2 i}\left(v_{4} x^{i}\right)=\left\{\begin{array}{l}
-v_{1} v_{2} v_{4} x^{c-1-i}(0 \leq i<a) \\
v_{4} x^{a+c-1-i}\left(a \leq i<\frac{a+c}{2}\right),
\end{array}\right. \\
& \cup x^{m-2 a-2 i}\left(v_{1} v_{2} x^{i}\right)=\left\{\begin{array}{l}
-v_{1} v_{2} v_{3} v_{4} x^{-a+c-1-i}(0 \leq i<-a+c) \\
v_{1} v_{2} x^{-a+2 c-1-i}\left(-a+c \leq i<\frac{-a+2 c}{2}\right),
\end{array}\right. \\
& \cup x^{m-(a+c)-2 i}\left(v_{1} v_{3} x^{i}\right)=v_{1} v_{3} x^{c-1-i}, \\
& \cup x^{m-(a+c)-2 i}\left(v_{1} v_{4} x^{i}\right)=v_{1} v_{4} x^{c-1-i}, \\
& \cup x^{m-(a+c)-2 i}\left(v_{2} v_{3} x^{i}\right)=v_{2} v_{3} x^{c-1-i}, \\
& \cup x^{m-(a+c)-2 i}\left(v_{2} v_{4} x^{i}\right)=v_{2} v_{4} x^{c-1-i}, \\
& \cup x^{m-2 c-2 i}\left(v_{3} v_{4} x^{i}\right)=v_{3} v_{4} x^{a-1-i}, \\
& \cup x^{m-(2 a+c)-2 i}\left(v_{1} v_{2} v_{3}\right)=v_{1} v_{2} v_{3} x^{-a+c-1-i}(a<c) \text {, } \\
& \cup x^{m-(2 a+c)-2 i}\left(v_{1} v_{2} v_{4}\right)=v_{1} v_{2} v_{4} x^{-a+c-1-i}(a<c) \text {, }
\end{aligned}
$$

whose linear combination can not be zero in cohomology. Thus $M$ is projective (c)-Lefschetz.

A nilpotent space is said to be formal if there is a quasi-isomorphism from its Sullivan minimal model to its rational cohomology algebra thought of as a DGA with zero differential [15]([5]). For example, compact Kähler manifolds are formal [4]. Finally we give a non-formal example.

Theorem 3.7. Let $M$ be a simply connected 16-dimensional manifold such that $\mathcal{M}(M)=\left(\Lambda\left(v_{1}, v_{2}, v_{3}, v_{4}\right), d\right)$ with $\left|v_{1}\right|=\left|v_{2}\right|=3,\left|v_{3}\right|=\left|v_{4}\right|=5, d\left(v_{1}\right)=$ $d\left(v_{2}\right)=0, d\left(v_{3}\right)=v_{1} v_{2}$ and $d\left(v_{4}\right)=0$. Then $M$ is projective non-Lefschetz.

Proof. There are only two cases for which $P\left(E^{k}\right)$ is c-symplectic.

First, let $D(y)=v_{1} v_{4} x^{i}+v_{2} v_{3} x^{i}+x^{i+4}$ with $|y|=7+2 i$. Then

$$
\operatorname{dim} P\left(E^{k}\right)=22+2 i \text { and } m=11+i .
$$

Then $P\left(E^{k}\right)$ is c-symplectic from $\left[x^{11+i}\right]=-\left[v_{1} v_{2} v_{3} v_{4} x^{i+3}\right] \neq 0$. But $P\left(E^{k}\right)$ does not have the Lefschetz property since $\left[v_{1} x^{8+i}\right]=\left[v_{1}\left(-v_{1} v_{4} x^{i}-v_{2} v_{3} x^{i}\right) x^{4}\right]$ $=-\left[v_{1} v_{2} v_{3} x^{i+4}\right]=\left[v_{1} v_{2} v_{3}\left(v_{1} v_{4} x^{i}+v_{2} v_{3} x^{i}\right)\right]=0$. 
Secondly, let $D(y)=v_{1} v_{2} v_{3} v_{4} x^{i}+x^{i+8}$ with $|y|=15+2 i$. Then

$$
\operatorname{dim} P\left(E^{k}\right)=30+2 i \text { and } m=15+i .
$$

Then $P\left(E^{k}\right)$ is c-symplectic from $\left[x^{15+i}\right]=-\left[v_{1} v_{2} v_{3} v_{4} x^{i+7}\right] \neq 0$. But $P\left(E^{k}\right)$ does not have the Lefschetz property since $\left[v_{1} x^{12+i}\right]=\left[v_{1}\left(-v_{1} v_{2} v_{3} v_{4} x^{i}\right) x^{4}\right]=$ 0 .

Note that the manifold $M$ of Theorem 3.7 is the product of $S^{5}$ with the pullback of the sphere bundle of the tangent bundle of $S^{6}$ by the canonical degree 1 map $S^{3} \times S^{3} \rightarrow S^{6}$. It is not formal since $H^{*}(M ; \mathbb{Q})$ contains an indecomposable element $\left[v_{1} v_{3}\right]$ (or $\left[v_{2} v_{3}\right]$ ), which corresponds to a non-trivial Massey product $\left\langle v_{1}, v_{2}, v_{1}\right\rangle$ (or $\left.\left\langle v_{2}, v_{1}, v_{2}\right\rangle\right)$ [4]. Recall that $Y=\left(S^{3} \times S^{8}\right) \sharp\left(S^{3} \times\right.$ $\left.S^{8}\right) \times S^{5}$ is formal and has the same rational cohomology as $M$. From Corollary 2.2 , we see that $Y$ is projective non-Lefschetz.

Remark 3.8. We know that $S^{3} \times S^{3} \times S^{5} \times S^{5}$ is projective (5)-Lefschetz from Theorem 3.6. It has the same rational homotopy groups as the manifold $M$ of Theorem 3.7. Thus projective Lefschetzness is not determined by the rational homotopy groups.

\section{References}

[1] R. Body, M. Mimura, H. Shiga, and D. Sullivan, p-universal spaces and rational homotopy types, Comment. Math. Helv. 73 (1998), no. 3, 427-442.

[2] R. Bott and L. Tu, Differential Forms in Algebraic Topology, GTM 82, Springer, 1982.

[3] G. R. Cavalcanti, The Lefschetz property, formality and blowing up in symplectic geometry, Trans. Amer. Math. Soc. 359 (2007), no. 1, 333-348.

[4] P. Deligne, P. Griffith, J. Morgan, and D. Sullivan, Real homotopy theory of Kähler manifolds, Invent. Math. 29 (1975), no. 3, 245-274.

[5] Y. Félix, S. Halperin, and J. C. Thomas, Rational Homotopy Theory, Graduate Texts in Mathematics 205, Springer-Verlag, 2001.

[6] Y. Félix, J. Oprea, and D. Tanré, Algebraic Models in Geometry, GTM 17, Oxford, 2008.

[7] M. Fernández, V. Muñoz, and L. Ugarte, Weakly Lefschetz symplectic manifolds, Trans. Amer. Math. Soc. 359 (2007), no. 4, 1851-1873.

[8] P. Hilton, G. Mislin, and J. Roitberg, Localization of Nilpotent Groups and Spaces, North-Holland Publishing Co., Amsterdam-Oxford; American Elsevier Publishing Co., Inc., New York, 1975.

[9] J. Kedra, KS-models and symplectic structures on total spaces of bundles, Bull. Belg. Math. Soc. Simon Stevin 7 (2000), no. 3, 377-385.

[10] G. Lupton and J. Oprea, Symplectic manifolds and formality, J. Pure. Appl. Algebra. 91 (1994), no. 1-3, 193-207.

[11] D. McDuff, Examples of simply-connected symplectic non-Kählerian manifolds, J. Differential Geom. 20 (1984), no. 1, 267-277,

[12] M. Mimura, G. Nishida, and H. Toda, Localization of $C W$-complexes and its applications, J. Math. Soc. Japan 23 (1971), 593-624.

[13] J. Oprea, The propagation of non-Lefschetz type, the Gottlieb group and related questions, J. Fixed Point Theory Appl. 3 (2008), no. 1, 63-77.

[14] J. Sato and T. Yamaguchi, Pre-c-symplectic condition for the product of odd-spheres, J. Homotopy Relat. Struct. 8 (2013), no. 1, 13-34. 
[15] D. Sullivan, Infinitesimal computations in topology, Inst. Hautes Études Sci. Publ. Math. 47 (1977), 269-331.

[16] A. Tralle, Homotopy properties of closed symplectic manifolds, Univ. Iagel. Acta Math. 38 (2000), 105-128.

[17] A. Tralle and J. Oprea, Symplectic manifolds with no Kähler structure, Lecture Notes in Mathematics, 1661. Springer-Verlag, Berlin, 1997.

[18] A. Weinstein, Fat bundles and symplectic manifolds, Adv. in Math. 37 (1980), no. 3, $239-250$.

HiRokAZU NishinOBU

KOCHI UNIVERSITY

2-5-1, AKeBOnO-CHO, KoCHI, 780-8520, JAPAN

E-mail address: cosmo51mutta@yahoo.co.jp

TOSHIHIRO YAMAGUCHI

KOCHI UNIVERSITY

2-5-1, AKeBono-CHO, KoCHI, 780-8520, JAPAN

E-mail address: tyamag@kochi-u.ac.jp 